\title{
Ethics and Subjectivity as equiprimordial values in Medicine
}

\author{
Ética e Subjetividade como valores \\ equiprimordiais em Medicina
}

\author{
Luis Antonio Proença Duarte Madeira \\ Universidade de Lisboa. Portugal. luismadeiramd@gmail.com
}

The exercise of medicine is particularly challenging in that, both in form and in content, it demands of us intricate competencies that can only be articulated if one masters various knowledge and sciences. In form, the medicine works with various "methods," valuing a multitude of contributions from the natural sciences, themselves having unpredictable progress and revolutions, but coming up against the "symptom" and the "sign" that are (and probably always will be) translated and thus intangible in their totality by whoever tries to grasp them. An example of this is the Encounter as the paradigm of accessing the Other - the moment in which another can offer himself (with greater or lesser complexity) to be understood in the certainty that we will only access him in this historical moment (space and time). In form, medicine is ontologically limited in certainty - not being able to know everything about someone is not a problem of ignorance about that person but the constituent of that element in the world being a person. In content, the fact that Human Beings have several layers of meanings of their experiences motivating hardships in objectifying suffering, help-seeking, and recovery - all of them aims of medical practice. First, each apparent fact, which seems to assure us the certainty with which we work, is only probabilistic in its meaning and consequence, as well as each medical action is indexed to the state of the art of our knowledge. What is valid today may not be valid tomorrow under other conditions and other circumstances. Second, each event gravitates an existential dimension vested in a complex system of values - our own values, the values of the person we are caring and the values of those who share a life with him (family, friends). Any simplistic approach risks the natural incomprehension of the interaction of "moral strangers" or going down two slippery slopes that touch each other and in which we always in - that of excessive paternalism or that of imbalanced respect of autonomy.

Besides these ontological aspects, several others rise from our historicity - demands are made of doctors today that are not easy to fulfill. For example, that they assume responsibility in actions that transcend humanity as it is usually understood - turning off ventilators, carrying out euthanasia, or assisting suicide - so that in their praxis, they are led to encounter transgressions of their morality by challenges where no 
preferable moral alternative can be found. Second, never before have we had so much desire to navigate autonomously in the uncertainty of the vital project (demanding respect for our autonomy) also never before have we asked others (especially doctors) so much to reduce the risk of being alive (read as being able to be cared for in a "medicalization of risk"). Third, we live in the shadow of the Brain Age, enacted, if not by others, by George Bush in the 1990s - and to a neuro-reductionism by cognitive neuropsychiatry and basic neuroscience. That has impacted practice from an epistemic point of view (Neuroeconomics, Neuromarketing, Neurotheology, Neuro-culture, Neuropolitics, etc.) and an ontological perspective on the gap between mind and brain, paradoxically, to the idea that 'we are our brains.' Here, neuroethics has had an apogee, yet new extraordinary paradigms were raised that lacked any integration with subjectivity and intersubjectivity in its rapid development.

More recently, and in counter-current, we were brought to the idea of "Ecology" of the brain with its emergent properties, the need to consider other preferable levels of explanation, and attention to mereological fallacies. This movement from the brain first in the direction of the individual (and his body) and then into the surrounding environment was fundamental to validate the limits of explanation again, re-subjectify the nature of experience, and include explanations both inter-subjectivity and context. It is now becoming ever clearer that we are immersed in the world and surrounded by others, and this is fundamental to any understanding of healthy and pathological behavior and determining medical interventions. That has had vast implications even for our respect for the world - whether in new environmental health facets or for the spaces of medical practice and recovery from illness in healthcare. Thus, our ethical project cannot distance itself from listening to our body, context, and the others we live with and respecting them holistically when organismically building our relationships. In such a sense, an embodied and immersed understanding of subjectivity is paramount to (and also nourished by) our personal and professional ethical projects in which the silence of one towards the other seems to hinder any true future for medical practice. 